\title{
How Do We "Raise" Ethically Minded Computer Students?
}

\section{Dr. Elizabeth Milonas, NYC College of Technology - City University of New York}

Elizabeth Milonas is an Assistant Professor with the department of Computer Systems at New York City College of Technology - City University of New York (CUNY). She currently teaches relational and nonrelational database theory and practice to undergraduate students in the Computer Systems Major at the college. In her courses, she incorporates ethical topics related to computers. She believes that by raising awareness of the impact technology can have on society, students are better prepared to assume an ethical and technical role in the business environment. Prior to her academic position, Dr. Milonas worked as a database administrator where she realized that technical expertise is only part of the skill set needed to succeed in a business setting. Her research focuses on the mechanisms used to organize big data in search result pages of major search engines. In addition, she is conducting research related to techniques for incorporating ethics in computer curriculum specifically in data science curriculum. 


\section{How Do We "Raise" Ethically Minded Computer Students?}

\section{Abstract}

Intelligent technology is increasingly being woven into the fabric of everyday life. It is becoming more and more a seemingly necessary and somewhat trusted component of society for both personal and non-personal day-to-day interactions. Developing such intelligent systems requires technical expertise, such as an in-depth knowledge of natural language processing or machine learning. However, in addition to technical expertise, a deep awareness and understanding of ethics and societal impact are also essential. Mastering knowledge of ethics and societal impact falls on the shoulders of computer professionals and programmers, whose role is to design and implement the decision-making component of intelligent systems. The development of intelligent systems with embedded ethical and social awareness is of paramount importance as a lack of such awareness has biased or unethical consequences. Such consequences were recently demonstrated when an algorithmic decision-making system at Amazon.com disqualified female job candidates. Preparing computer students to meet the demand of intelligent technology implementation, requires incorporating the topics of ethics and societal impact into computer curriculum. These topics should be introduced and reinforced throughout the computer curriculum, beginning at the introductory courses, and continuing to the advanced courses. This method ensures that computer students acquire the necessary technical and ethical skills needed. The combination of these skills ensures effective design, implementation and deployment of intelligent systems that are both technologically advanced and ethically mindful.

\subsection{Introduction}

During the past few decades, the world has changed as a result of advances in technology [1],[2],[3]. These advances in technology have impacted and transformed society both on a personal and professional level. They have seemingly improved the quality of life [1],[2],[3] by providing a means of knowledge acquisition and exchange, and by connecting people and businesses everywhere. Most of today's advanced technologies are powered by machine learning algorithms. The machine learning algorithms examine trends in data and form relationships by recognizing the patterns in the data. Once the relationships are established, machine learning algorithms use these relationships to solve complex problems [4],[5]. Examples of machine learning in daily lives abound. Virtual personal assistants, such as Siri or Alexa, use machine learning algorithms to collect usage data and refine the data to provide a more accurate and personal experience for the user [8]. Machine learning algorithms are used in online transportation networks such as Uber or Lyft [9]. These algorithms use traffic information gathered from GPS navigation services, to estimate the price of a trip based on the time of day and traffic conditions [9]. Social Media platforms, such as Facebook and Instagram, use machine learning algorithms to customize the user experience. In such cases, the machine learning algorithms are used to personalize news feed and ads, and to provide a list of suggested friends or followers [7]. On many commercial websites, machine learning algorithms are used for online customer support. These algorithms, which are called "chatbots," provide the user with general information and are also used to answer basic questions [7]. Google and other search engines use machine learning algorithms to improve the search process and to recommend other searches 
related to the original search [10]. In shopping websites, machine learning algorithms are used to recommend items based on previous shopping history or buying trends [11]. In the medical field, machine learning algorithms are used to process information and recognize patterns from MRI or mammogram test results [6]. They are also used to predict variables that can potentially prevent medical conditions, such as diabetes [6]. Corporations use machine learning algorithms to screen potential job applicants [24]. The financial sectors use machine learning algorithms to determine trends in spending and personalize offers to customers [7]. In addition, these algorithms are used to predict risk. Here machine learning algorithms examine credit history and other financial data to determine if individuals qualify for personal loans and mortgages [7].

Machine learning algorithms are vital in the development of present-day technologies. It is predicted that their importance will increase exponentially as the demand for new and more advanced intelligent technologies increases. In the near future, it is anticipated that machine learning algorithms will be used to improve conversational artificial intelligent systems (chatbots) [13], driverless cars, voice commands and queries [14], epidemic and outbreak modeling, wildlife preservation, conflict resolution [15], real-time traffic monitoring, and locating missing persons and stolen vehicles [16]. It is also predicted that machine learning algorithms will become increasingly accessible not only to the large enterprises, but to all businesses [17]. Currently, machine learning algorithms are ubiquitous and can affect lives. They are increasingly being used in more of the decision-making processes such as, determining which applicants are qualified for loans or choosing qualified job applicants [23, p. 231]. It is therefore important that the computer professionals and programmers, who design and implement these technologies, possess not only the technical sophistication required for the task, but also a deep understanding of ethical and societal impact. It is this combination of technical knowledge and ethical awareness, that can prevent bias and ensure the inclusion of people and groups of people [25], [30]. In the past, the designers or manufacturers of computer systems were seldom involved in the ethics of the technology as this responsibility was left to the user of that technology [30]. However, in intelligent technology, the decision-making process is part of the algorithm. Computer professionals and programmers are therefore tasked with considering the possible ethical impact of an algorithm at the design and coding stages [30]. Oftentimes, computer professionals and programmers, although technically savvy, may not be aware of the ethical dilemmas related to the algorithm [30]. Such examples already exist in today's society and will grow exponentially, as these algorithms increasingly become part of the day-to-day decisionmaking processes. Currently in some states, prison models are used to reduce prejudice in sentencing [23, p. 25]. The algorithms that drive these models are based on answers to survey questions regarding crime involvement. However, these surveys contain questions that are biased [23, p.27]. For example, a prisoner is asked if he/she or any members of his/her family, friend group, or any acquaintance, has had any contact with the police [23, p. 25]. Such a question is inherently biased as statistically speaking, young black males are more likely to have been stopped by police than young white males [23, p. 25]. Also, statistically speaking, convicted criminals raised in poor and troubled neighborhoods are more likely to know people who have had some contact with the police [23, p. 26]. In some states, judges use these prison models to determine the length of a prison sentence [23, p. 27]. However, statistics have shown that prisoners who are given longer sentences are exposed to other criminals for a longer time, increasing their likelihood of returning to prison after being released [23, p. 27]. Statistics also 
indicate that criminals with longer sentences have less of a chance of finding jobs and ultimately increasing their likelihood of returning to criminal activity and eventually prison [23, p. 27].

In the same manner, employers are using hiring models to help determine potential candidates for a job [23, p. 7]. One such model uses credit scores to evaluate new hires. The idea behind this model is that, if people pay their bills on time, then they are more likely to come to work on time and follow the rules [23, p.7]. However, making the connection between low credit scores and responsibility is biased. Many uncontrollable factors can contribute to low credit scores such as illness or loss of income [23, p.7]. Unfortunately, the use of such biased hiring algorithms decreases the likelihood of someone with a low credit score obtaining a position, even if the individual is responsible and a good candidate for the job [23, p. 7]. This creates a vicious cycle. Joblessness can result in further economic hardship. Economic hardship can further worsen credit scores. Having bad credit scores makes it even more difficult to get hired for a job [23, p.7]. Amazon is an example of a corporation that uses hiring models to determine potential job candidates. Recently the Amazon hiring algorithm displayed bias when it excluded female applicants [34]. This hiring algorithm determined candidates by examining the patterns in resumes submitted to Amazon over a period of ten years [34]. However, during this period, a very high percentage of the job applicants were male, due to the prevalent computer industry trend at this time [34]. Using this observed trend for identifying good job candidates, the algorithm gave a high score to male applicants and penalized female applicants. As a result, female applicants were excluded altogether from the results [34]. These situations highlight the fact that no model can include the complexity inherent in society [23, p. 20]. These models are not programmed to be mindful of the social and ethical nuances which matter greatly and ultimately make a difference in the outcome [23, p. 27]. Removing bias from algorithms requires the algorithm to be programmed not only to be technically efficient, but also ethically conscious [23, p.20]. This difficult task falls on the shoulders of computer professionals and programmers as models are nothing more than an "abstract representation of some process" [23, p. 18]. Computer professionals and programmers have to be able to make choices [23, p. 204] and be the gatekeepers of ethical issues such as bias, ensuring that no one is left behind [39]. Hence, it is paramount that computer curricula embed ethics and societal issues related to bias [35] to provide computer students with a deeper understanding of the ethical dilemmas associated with algorithm design and coding [30]. Ultimately, such an understanding will help in the process of identifying possible methods for addressing ethical concerns, such as bias in algorithm design and implementation [33]. The production of more ethically conscious algorithms, will help in reducing the potential negative impact of such technologies on society[33].

This paper is divided into several sections. In section 2.0 of this paper, literature related to machine learning is reviewed. A synthesis of the issues related to this domain are outlined. Specifically, a discussion of the importance of incorporating ethics in computer curriculum including algorithm bias is presented. Section 3.0 of this paper outlines a plan for raising awareness of algorithm bias in a newly developed Introduction to Data Science course. Subsections 3.1 through 3.4 detail the plan by indicating the goal, identifying the learning outcomes, discussing the class activities, and proposing an evaluation strategy as well as a strategy for presenting the results. The last section of this paper discusses the future study.

2.0 Related work 
Machine learning is defined as a system that mimics human learning by acquiring knowledge from the real world and performing based on this knowledge [18]. Unlike humans, machine learning algorithms cannot reason [18]. They cannot include the subtleness of human interaction or the complexity that exists in the world [23, p. 20]. Important information will inevitably be missing from these machine learning algorithms [23, p. 20]. These algorithms have inherent bias as they reflect the judgments, good or bad, of their designers and programmers [23, p. 21], and in some cases, process data that is itself biased [12]. This makes it difficult for machine learning algorithms to produce fair results[12]. Ultimately though, the responsibility of ensuring fairness in the data generated by these algorithms falls on the shoulders of computer professionals and programmers. These computer professionals and programmers are the gatekeepers, ensuring no one or no group of people is excluded [12]. Therefore, of paramount importance is educating the future computer professional and programmer in not only the technical skills needed to design and code machine learning algorithms, but also the ethical concerns and dilemmas related to these algorithms.

\subsection{Importance of the Inclusion of Ethical Topics in Computer Curriculum}

Quinn [26] emphasizes the importance of including ethical issues in each computer course throughout the computer curriculum. He [26] states that this method targets specific ethical issues relevant to specific computer topics. For example, ethical issues related to database technologies are discussed in a database course, likewise, ethical issues related to computer security are discussed in a computer network and security course [26]. Metcalf et al. [27] also agree with Quinn in dispersing ethical topics throughout the computer curriculum and in each related computer course. These researchers [27] state that an integrative approach is more effective especially if accompanied by relevant case studies that outline the ethical dilemma and possible solutions [27]. Likewise, Jones [28] believes that to bring awareness to key ethical issues, these issues should be discussed in every computer course throughout the curriculum. Jones[28] suggests that it is important that students learn the technical aspects of the computer topic and the ethical issues related to that topic. Like Quinn [26], Jones [28] suggests the use of ethics-related projects tailored to the computer topic covered. Chowdhury [29] also agrees with Quinn [26], Metcalf et al. [27], and Jones [28], and suggests embedding ethical and moral issues throughout the computer curriculum. Chowdhury [29] recommends the use of role-play, drama, simulation, educational games, debates, discussions, projects, group work and other such interactive and group-related activities to reinforce ethical concepts. Sirpan et al. [31] emphasize it is more effective to teach ethics throughout the computer curriculum by infusing workshops, team projects, individual assignments and soliciting guest speakers, to help computer students understand the ethical dilemmas. Polmear et al. [32] outline the efficiency of incorporating ethical themes throughout the computer curriculum. Polmear et al. [32] indicate that this method is most effective. They [32] state that most computer curricula in undergraduate programs have too many required computer courses to be able to accommodate an additional computer ethics course [32]. Infusing ethical issues and dilemmas in each computer course throughout the computer curriculum allows students to be continually exposed to key ethical issues [38]. The persistent presence of key ethical issues throughout the curricula reinforces the importance of these issues [38]. It encourages students to see ethics as an integral component in the computer domain [38]. All the researchers [26],[27],[28],[29],[31],[32],[38] highlight the importance of 
integrating ethical concepts with computer skills. The goal for such integration is to create a complete education for computer programmers that includes not only technical proficiency but ethical awareness.

\subsection{Importance of the Inclusion of Algorithm Bias as an Ethical Topic in the Computer Curriculum}

In terms of machine learning algorithms, bias is the prevalent ethical theme found in a majority of the literature. In the domain of machine learning, bias is defined as the "systematic unfairness perpetrated on individuals or groups [22]." As technology becomes more advanced, especially in autonomous systems such as decision-making systems, ethical framework and values become imperative in the design and coding of machine learning algorithms [21]. It is important that within the design process, computer professionals keep mindful of human values and separate the design from biased values [21]. According to Friedman [22], bias is one of the key ethical issues which computer professionals and programmers need to address when designing algorithms. Friedman [22] states that it is imperative to include human values, such as those related to eliminating bias, in a "principled and comprehensive" way in every aspect of design. These human values are as important in design as are usability, reliability, and correctness [36]. Friedman [22] emphasizes that it is necessary to add human values to the design of algorithms early on and throughout the design process.

The pressing need for bias awareness and understanding in the design and results of machine learning algorithms is outlined by Wang[19] who analyzed the results of a survey of 590 participants using machine learning algorithms. The results of this study showed [19] that awareness of algorithm bias increases with the amount of education that the computer professional received. The study results also showed that both general education and computer science education influences perceptions of bias and fairness [19]. Wang [19] states that people with greater computer knowledge have a better understanding of the type of information that an algorithm should generate. In addition, people with greater computer knowledge have a better understanding of how this information can be processed fairly. The findings from this study [19] suggests that computer education specifically related to algorithms, improves awareness of algorithm bias and fairness. Higher education curricula also reflect the importance of incorporating topics related to bias in machine learning algorithms. An examination of the syllabi of 115 "tech ethics" courses from various universities [41], identified bias as the common and recurring ethical theme being taught. The study results [41] indicate that the theme of bias is infused in many of the ethical categories related to technology. These categories include privacy, security and ownership [20].

\subsection{Plan of Action - Raising Awareness of Algorithm Bias in an Introduction to Data Science Course}

Awareness of bias is paramount in the design and development of algorithms as has been shown in this paper. In this section a plan for implementing awareness and understanding of algorithm bias in a newly developed Introduction to Data Science course will be described. This plan is grounded in theory, methods and recommendations discussed in section 2.0, and includes best methods for incorporating this topic as well as learning outcomes. 


\subsection{The goal and learning outcomes}

The primary goal of this initiative is to help undergraduate computer majors become cognizant of the fact that machine learning algorithms can potentially generate bias, and that such bias can exclude individuals or groups of people. It is important for these students to gain such an awareness as they will ultimately be the designers and coders of these machine learning algorithms. This goal can be broken down into the following learning outcomes [37],[40],[41]:

- Students will identify the ethical issue of bias in machine learning algorithms

- Students will identify the ethical dilemmas associated with algorithm bias including the various standpoints and the possible consequences

- Students will identify their responsibility as future computer professionals

- Students will identify the prescribed ethical standards set by institutions such as ACM/IEEE or ABET

\subsection{Class Activities}

Class activities will include case studies, weekly responses, discussions, guest lecture series and team projects. These methods have been proven effective in raising awareness and encouraging an understanding of ethical issues [29],[31].

1. Analysis of real-world cases both past and present related to algorithm bias will be assigned. Students will work in teams to discuss the implications of the bias including the issues, the effects, the recipients, the far-reaching results and other ethical dilemmas related to bias. Students will also be asked to use role-play to identify the main biasrelated issues and concerns related to the case study.

2. Weekly responses to topics related to specific algorithm bias found in relevant readings, TedTalks, and news articles, etc. will be assigned to broaden students' understanding of the effects of bias in today's society.

3. Current events related to ethics on the whole and/or bias specifically will be posted on an online discussion board. Students will be encouraged to participate online by providing their insight related to the current event.

4. Guest lecturers/speakers will be invited to discuss the ethical concerns related to algorithm bias. They will be randomly chosen from a group of computer professionals. These computer professionals are members of an Advisory Board that guides and informs the computer department on computer-related issues in the workforce.

5. A team project will be assigned which will identify a machine learning algorithm. The team will be responsible for outlining the function of the algorithm as well as its effects. Students will refer to prescribed industry standards. Students will identify the ethical bias inherent in the algorithm and detail the ethical dilemmas which will ensue as a result of the algorithm. Students will also be asked to identify the recipients and outline the farreaching effects of the algorithm. Students will have to identify their responsibility as designers and coders. The results will be presented in class via PowerPoint presentation. A report will also be submitted.

6. Quizzes and Exams will be given to reinforce the concepts discussed. 
Table 1 below displays the correlation between the learning outcomes and the class activity.

\begin{tabular}{|l|l|}
\hline Learning Outcomes & Class Activities \\
\hline $\begin{array}{l}\text { Identify the ethical issue of } \\
\text { bias in machine learning } \\
\text { algorithms }\end{array}$ & $1,2,3,4,5,6$ \\
\hline $\begin{array}{l}\text { Identify the ethical dilemmas } \\
\text { associated with algorithm } \\
\text { bias including the various } \\
\text { standpoints and the possible } \\
\text { consequences }\end{array}$ & $1,2,3,4,5,6$ \\
\hline $\begin{array}{l}\text { Identify student responsibility } \\
\text { as future computer } \\
\text { professionals }\end{array}$ & $4,5,6$ \\
\hline $\begin{array}{l}\text { Identify the prescribed ethical } \\
\text { standards set by institutions } \\
\text { such as ACM/IEEE or ABET }\end{array}$ & 5,6 \\
\hline
\end{tabular}

Table 1: Learning outcomes and the related class activity

\subsection{Evaluation}

\subsubsection{Student}

The students will be asked to evaluate the course using the college evaluation form. In addition, students will be asked to complete a survey specific to the ethics component of the course. Questions regarding the usefulness of bias-related topics covered in the course and the effectiveness of the teaching methods will be asked. Also, students will be asked to give suggestions regarding additional bias-related topics which can be relevant to the course. Students will also be asked to volunteer to participate in focus groups.

\subsubsection{Advisory Board}

A survey will be given to Advisory Board members to solicit their expert opinions related to bias topics covered in the course. Advisory Board members will be asked to rate the ethical topics covered in the course. They will also be asked for their input regarding the addition of other bias-related topics which they feel are important in today's tech industry.

\subsection{Results of student and Advisory Board surveys and focus groups}

Statistical analysis of the student and Advisory Board surveys as well as the learning outcomes will be conducted. Content analysis of focus group discussions will also be conducted.

\subsection{Future Direction}


The quantitative and qualitative findings from the surveys and focus groups will be analyzed, learning outcomes will be measured and these findings will be presented in a future paper. Based on these findings and if warranted, revisions will be made to the course outline, outcomes and/or activities. The ethical component related to bias in the course will continue to be evaluated in the next offering of the class and surveys, focus groups and evaluation of learning outcomes will ensue. Quantitative and qualitative results gathered from the second offering of the class will be compared and contrasted with previous results and the findings will be reported in a future paper.

\section{Work Cited}

[1] W. De Wet, E. Loekemoer and J.A. Nel, "Exploring the impact of information and communication technology on employees' work and personal lives," SA Journal of Industrial Psychology, vol. 42, ed. 1, Jun. 2016.

[2] P. Hanafizadeh, S. Ghandchi and M Asgarimehr, "Impact of Information Technology on lifestyle: A literature review and classification," International Journal of Virtual Communities and Social Networking, vol. 9, is. 2, Apr.-Jun. 2017.

[3] S. Deb, "Information technology, its impact on society and its future," Advances in Computing, vol. 4, is. 1, pp. 25-9, 2014.

[4] D. J. Fuchs, "The dangers of human-like bias in machine-learning algorithms," Missouri S\&T's Peer to Peer, vol. 2, is. 1, May 2018.

[5] B. J. Erickson, P. Korfiatis, Z. Akkus, and T. L. Kline, "Machine learning for medical imaging," RadioGraphic, vol. 37, no. 2, pp. 505-15, Mar.-Apr. 2017

[6] B. Marr, "The top 10 AI and machine learning use cases everyone should know about," forbes.com, Sep. 2016. [Online]. Available:

https://www.forbes.com/sites/bernardmarr/2016/09/30/what-are-the-top-10-use-cases-formachine-learning-and-ai/\#5f190acc94c9 [Accessed: January 3, 2020].

[7] N. Heinrich, "What is machine learning? A quick guide to basic concepts," builtin.com, Jan. 2020. [Online]. Available: https://builtin.com/artificial-intelligence/what-is-machine-learningbasic-concepts [Accessed: January 10, 2020].

[8] B. Barrett, “The year Alexa grew up. Amazon's voice assistant made considerable in 2018 through the continued refinement of machine learning techniques," wired.com, Dec. 2018. [Online]. Available: https://www.wired.com/story/amazon-alexa-2018-machine-learning/ [Accessed: January 3, 2020].

[9] J. Msv, "Managing machine learning models the Uber way," forbes.com, Jun. 2019. [Online]. Available: https://www.forbes.com/sites/janakirammsv/2019/06/26/managing-machine-learningmodels-the-uber-way/\#534b72624ae4 [Accessed: January 3, 2020]. 
[10] K. Rowe, "How search engines use machine learning: 9 things we know for sure," searchenginejournal.com, Feb. 2018. [Online]. Available:

https://www.searchenginejournal.com/how-search-engines-use-machine-learning/224451/ [Accessed: January 3, 2020].

[11] Z. Ruan and K. Siau, "Digital marketing in the artificial intelligence and machine learning age," in Proc. of the 25th AMCIS (The Americas Conference on Information Systems)

Conference, August 15-17, 2019, Cancun, Mexico [Online]. Available:

https://aisel.aisnet.org/cgi/viewcontent.cgi?article=1587\&context=amcis2019 [Accessed:

January 20, 2020].

[12] S. C. Olhede and P.J. Wolfe, "The growing ubiquity of algorithms in society: implications, impacts and innovations," Philosophy Transactions, Jun. 2018. [Online]. Available:

https://royalsocietypublishing.org/doi/pdf/10.1098/rsta.2017.0364

[Accessed: January 20, 2020].

[13] M. Yao, "What are important AI and machine learning trends for 2020?," Forbes.com, Jan. 2020. [Online]. Available: https://www.forbes.com/sites/mariyayao/2020/01/22/what-are-important-ai--machine-learning-trends-for-2020/\#b0fe65d23239 [Accessed: January 10, 2020].

[14] M. Deer, "Seven important predictions for machine learning in 2020," towardsdatascience.com, Dec. 2019. [Online]. Available: https://towardsdatascience.com/sevenimportant-predictions-for-machine-learning-in-2020-ca840826e25d [Accessed: January 10, 2020].

[15] "15 social challenges AI could help solve," forbes.com, Sep. 2019. [Online]. Available: https://www.forbes.com/sites/forbestechcouncil/2019/09/03/15-social-challenges-ai-could-helpsolve/\#fab5a1a3533d [Accessed: January 10, 2020].

[16] B. Marr, "5 important Artificial Intelligence predictions (for 2019) everyone should read," forbes.com, Dec. 2018. [Online]. Available:

https://www.forbes.com/sites/bernardmarr/2018/12/03/5-important-artificial-intelligence-

predictions-for-2019-everyone-should-read/\#5ba301ba319f [Accessed: January 10, 2020].

[17] G. Press, “120 AI Predictions for 2020,” forbes.com, Dec. 2019. [Online]. Available: https://www.forbes.com/sites/gilpress/2019/12/09/120-ai-predictions-for-2020/\#5caa71f50cf9 [Accessed: January 18, 2020].

[18] L. Portugal, P. Alencar, and D. Cowan, "The use of machine learning algorithms in recommender systems: a systematic review," Expert Systems with Applications, 2018. [Online]. Available: https://arxiv.org/pdf/1511.05263.pdf\%20(https://arxiv.org/pdf/1511.05263.pdf) [Accessed: January 12, 2020].

[19] R. Wang, F. M. Harper and H. Zhu, "Factors influencing perceived fairness in algorithmic decision-making: Algorithm outcomes, development procedures, and individual differences," 
CHI '20, April 25-30, 2020, Honolulu, HI. [Online]. Available:

https://arxiv.org/pdf/2001.09604.pdf [Accessed: January 12, 2020].

[20] G. White and T. Ariyachandra, "Big data and ethics: Examining the grey areas of big data analytics," Issues in Information Systems, vol. 17, is. IV, pp. 1-7, 2016.

[21] S. Umbrello and A. F. DeBellis, "A value-sensitive design approach to intelligent agents," Artificial Intelligence Safety and Security, Jan. 2018. [Online]. Available:

file:///Users/emilonas/Downloads/SSRN-id3105597.pdf [Accessed: January 13, 2020].

[22] B. Friedman, P. H. Kahn Jr. and A. Borning, "Value sensitive design and Information Systems," in Human-Computer Interaction and Management Information Systems: Foundations Advances in Management Information Systems, Vol. 5, Ping Zhang and Dennis Galletta, Eds. New York: M. E. Sharpe, 2006, pp. 348-372.

[23] C. O’Neil, Weapons of Math Destruction. New York: Broadway Books, 2017.

[24] W.M.R.W. Ibrahim and R. Hassan, "Recruitment trends in the era of industry 4.0 using artificial intelligence: Pros and cons," Asian Journal of Research in Business and Management, vol.1, no. 1, pp.12-21, Aug. 2019.

[25] J.L. Leidner and V. Plachouras, "Ethical by design: Ethics best practices for natural language processing," in Proc. of the First Workshop on Ethics in Natural Language, Valencia, Spain, April 4th, 2017, pp. 8-18. [Online]. Available: https://www.aclweb.org/anthology/W171604.pdf [Accessed: January 21, 2020].

[26] M.J. Quinn, "On teaching computer ethics within a computer science department," Science and Engineering Ethics, vol. 12, is. 2, pp. 335-343, 2006.

[27] J. Metcalf, K. Crawford, and E. Keller, "Pedagogical approaches to data ethics," Data \& Society, Apr. 2015. [Online]. Available: https://bdes.datasociety.net/wpcontent/uploads/2016/10/Data-Ethics-Course-Summary.pdf [Accessed: January 30, 2020]

[28] S. Jones, "Doing the right thing: computer ethics pedagogy revisited," Journal of Information, Communication and Ethics in Society, vol. 14, is. 1, pp. 33-48, 2016.

[29] M. Chowdhury, "Emphasizing morals, values, ethics and character education in science education and science teaching," The Malaysian Online Journal of Educational Science, vol. 4, is. 2, 2016. [Online]. Available: https://files.eric.ed.gov/fulltext/EJ1095995.pdf

[Accessed: January 13, 2020].

[30] H-M. Jarvinen, "Ethics as a skill of software engineering," in Proc. of SEFI 2017 Annual Conference, Azores, Portugal, September 18-21, 2017, J.C. Quadrado, J. Bernardino, and J. Rocha, Eds. Brussels: European Society for Engineering Education SEFI, 2017, pp. 856-862. 
[31] M. Skirpan, N. Beard, S. Bhaduri, C. Fiesler, T. Yen, "Ethics education in context: A case study of novel ethics activities for the CS classroom," in Proc. of SIGCSE '18, Baltimore, MD, February 21-24, 2018. [Online]. Available:

https://cmci.colorado.edu/ cafi5706/sigcse2018_ethics.pdf [Accessed: January 10, 2020].

[32] M. Polmear, A.R. Bielefeldt, D. Knight, C. Swan, and N. Canney, "Faculty perceptions of challenges to educating engineering and computing students about ethics and societal impacts," 2018 ASEE Annual Conference and Exposition, Salt Lake City, Utah, June 24-27, 2018. [Online]. Available: file:///Users/emilonas/Downloads/faculty-perceptions-of-challenges-toeducating-engineering-and-computing-students-about-ethics-and-societal-impacts\%20(1).pdf [Accessed: January 10, 2020].

[33] E. Cech, "Culture of Disengagement in Engineering Education?," Science, Technology, \& Human Values, vol. 39, no. 1, pp. 42-72, 2013.

[34] J. Dastin, "Amazon scraps secret AI recruiting tool that showed bias against women," Reuters.com, Oct. 2018. [Online]. Available: https://www.reuters.com/article/us-amazon-comjobs-automation-insight/amazon-scraps-secret-ai-recruiting-tool-that-showed-bias-againstwomen-idUSKCN1MK08G [Accessed: January 10, 2020].

[35] T. S. Purewal Jr., C. Bennett, and F. Maier, "Embracing the social relevance: Computing, ethics and the community," SIGCSE '07, March 7-10, 2007, Covington, Kentucky. [Online]. Available: http://mars.umhb.edu/ wgt/cisc4370/p556-purewal.pdf [Accessed: January 20, 2020].

[36] B. Friedman2, P. H. Kahan Jr. and A. Borning, "Value sensitive design: Theory and Methods," University of Washington CSE Technical Report, February 12, 2001. [Online]. Available:

http://citeseerx.ist.psu.edu/viewdoc/download?doi=10.1.1.11.8020\&rep=rep1\&type=pdf [Accessed: January 12, 2020].

[37] C. Huff and W. Frey, "Moral pedagogy and practical ethics," Science and Engineering Ethics, vol. 11, Is. 3, 2005, pp. 389-408.

[38] M.E. Califf and M. Goodwin, "Effective incorporation of ethics into courses that focus on programming," SIGCSE '05, February 23-27, 2005, St. Louis, Missouri. [Online]. Available: http://www.cs.potsdam.edu/faculty/laddbc/Teaching/Ethics/StudentPapers/2005CaliffEffectiveIncorporationOfEthicsIntoCoursesThatFocusOnProgramming.pdf [Accessed: January 21, 2020].

[39] C. DeBrusk, "The risk of machine learning bias (and how to prevent it)," MIT Sloan Management Review, Mar. 2018. [Online]. Available:

https://www.oliverwyman.com/content/dam/oliverwyman/v2/publications/2018/november/Risks-of-machine-learning.pdf [Accessed: January 3, 2020].

[40] Naz, Embedding ABET's outcomes in a software engineering course," ASEE's $123 \mathrm{rd}$ Annual Conferences \& Exposition, New Orleans, LA, June 26-29, 2016. [Online]. Available: 
file:///Users/emilonas/Downloads/embedding-abet-s-outcomes-in-a-software-engineeringcourse.pdf [Accessed: January 12, 2020].

[41] C. Fiesler, N. Garrett, and N. Beard, "What do we teach when we teach tech ethics? A syllabi analysis," In the 51 st ACM Technical Symposium on Computer Science Education (SIGCSE '20), March 11-14, 2020, Portland, Oregon. [Online]. Available:

https://cmci.colorado.edu/ cafi5706/SIGCSE2020_EthicsSyllabi.pdf [Accessed: January 13, 2020]. 